\title{
RNA editing: an overlooked source of fine- scale adaptation in insect vectors?
}

\author{
Xavier Grau-Bové I* $^{*}$ and David Weetman ${ }^{{ }^{*}}$
}

6 1. Department of Vector Biology, Liverpool School of Tropical Medicine, Liverpool, UK

* Corresponding author: XGB (avier.graubove@ gmail.com), DW (avid.weetman@1stmed.ac.uk)

\section{Abstract}

RNA editing is a source of molecular diversity that regulates the functional repertoire of animal

10 transcriptomes. Multiple studies in Drosophila have revealed that conserved editing events can be a source of evolutionary adaptations, and there is a solid body of evidence linking editing and the

12 fine-tuning of neural genes, which are often targeted by insecticides used in vector control. Yet, despite these suggestive connections, genome-wide analyses of editing in insect vectors are

14 conspicuously lacking. Future advances will require complementing the growing wealth of vector genomes with targeted transcriptome analyses. Here, we review recent investigations of the genetic

16 footprints of adaptive RNA editing in insects and provide an overview of new methodologies applicable to studies of RNA editing in insect vectors.

18 Highlights

- RNA editing introduces transcript-specific mutations that are not detected in genetic assays.

20 - The regulated edition of transcriptomes is conserved and globally adaptive across various Drosophila species, suggesting a general principle in insects.

22 - RNA editing fine-tunes the functions of neural channels that are involved in insecticide resistance. Yet, genome- and transcriptome-wide studies in insect vectors are still lacking. 


\section{Introduction}

The synthesis of transcripts involves post-processing and chemical modifications of the RNA

26 molecules, which can fine-tune their functions and create distinct isoforms from a single DNA template. RNA editing is a form of transcript post-processing that involves the chemical

28 modification of single bases in immature RNA molecules, resulting in transcript-specific ribonucleoside mutations [1] $\square$. RNA editing is a source of molecular novelty that may fuel adaptive

30 evolution $[2,3] \square$, in common with other mechanisms of transcriptome diversification - with which it should not be confused - such as alternative splicing. RNA editing is regulated by conserved cis-

32 encoded signals $[1,3] \square$ that are subject to natural selection. Consequently, both the regulatory causes and the adaptive consequences of these transcriptomic mutations can be readily studied from

34 a population genomic perspective.

Yet surprisingly, there have been very few studies of RNA editing in insect vectors, and none

36 focusing on its population genetics. Here we review evidence of editing in disease vectors, in which it may generate functional changes in genes involved in adaptation to insecticide resistance. The

38 emergence of resistance is an important public health issue, as it jeopardises the effectiveness of vector control programmes. Genetic monitoring programmes of insecticide resistance, however, do

40 not routinely probe possible adaptations mediated by RNA editing. We also consider recent studies on the role of editing in environmental adaptations in model insects, primarily Drosophila

42 melanogaster, and its regulation via population-specific polymorphisms. Finally, we provide examples of genome-wide approaches on the interaction between microevolutionary processes and

44 RNA regulation that can inform future studies utilising vector genomic resources.

\section{The molecular basis of RNA editing}

46 Animals exhibit multiple types of RNA editing, each of them effected by a different family of enzymes that target specific nucleotides, and often show preference for certain types of transcripts

48 and sub-regions within transcripts (coding and non-coding). The most common type of editing is the deamination of adenosine into inosine (A-to-I) by ADAR enzyme family $[1,4] \square$, which is

50 conserved in most animals [5] $\square$. Inosines are recognised as guanosines by the translational machinery and the reverse transcriptase used in RNA sequencing protocols [1,3] $\square$, making A-to-I,

52 effectively, a transcript-specific A-to-G substitution. Insects also undergo other, less common [3] $\square$, types of editing: C-to-U deamination effected by the cytidine deaminase APOBEC-1 [6] $\square$, and U-

54 to-C or G-to-A trans-aminations [7] $\square$. 
RNA editing can have various effects at the molecular level (Figure 1) [1,3] $\square$. The most direct

56 consequences are 'recoding' changes, which is relatively common in Drosophila [8] $\square$, and can result in non-synonymous substitutions and possibly new protein isoforms (Figure 1a). Editing can

58 also influence alternative splicing: it can disrupt or create new cis-regulatory signals that regulate splicing (e.g. the acceptor/donor splice sites) [9] $\square$ (Figure 1b); alter the stability of the dsRNA

60 structures formed during splicing $[10,11] \square$; and the editing molecular machinery can compete with splicing factors for physical access to the nascent RNA $[10,11] \square$. A-to-I changes also regulate

62 microRNA activity (Figure 1c): editing of precursor mRNAs ( $3^{\prime}$ or $5^{\prime}$ untranslated regions) or the microRNA itself can reconfigure microRNA binding sites and influence transcript expression and

64 degradation rates $[1,12] \square$. Finally, ADAR enzymes also act on clustered editing sites located in repetitive pre-mRNA regions, often rich in retroelements such as Alu that are prone to form dsRNA

66 structures [3] $\square$. Intense editing of repetitive elements been linked to the regulation of the cytosolic immune response against dsRNA structures [3] $\square$, and to the exonisation of retroelements via

68 creation of new splicing sites $[1] \square$.

(a)
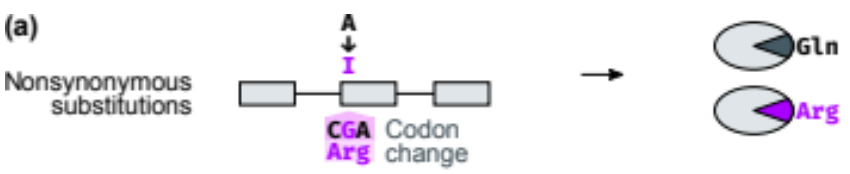

Protein substitutions
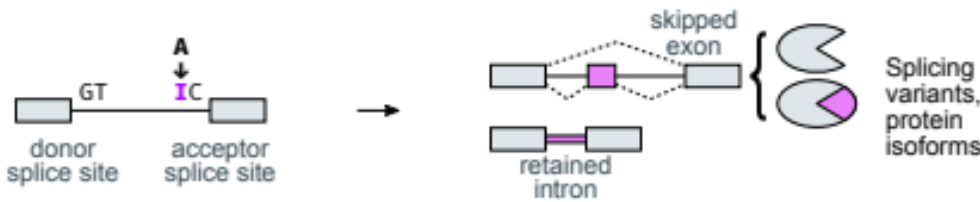

Splicing

(b) Alter splicing
regulatory sites splice site retained
intron protein isoforms

(c)
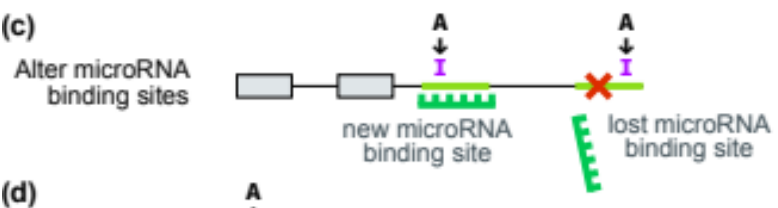
(d)
Alter microRNA binding sites
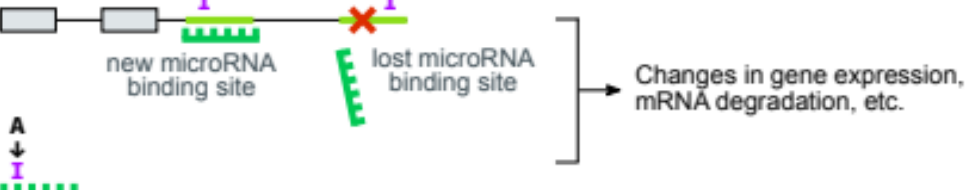

Figure 1. Molecular effects of RNA editing. (a) 'Recoding' events result in non-synonymous substitutions and the production of new protein isoforms. (b) Editing can modify conserved splicing regulatory signals present in precursor mRNA, such as donor (GT) or acceptor (AC) splice sites. (c) Editing can add/remove binding sites for microRNAs (often present in untranslated regions of the precursor mRNA), or (d) act on the microRNA molecule itself.

\section{Genetic footprints of adaptive RNA editing}

Transcript editing results in increased sequence diversity [3] $\square$, potentially providing a source of evolutionary adaptations [2] $\square$. RNA variants enable the exploration of phenotypic space (e.g. novel protein isoforms) that is inaccessible by genomic mutations, which can carry fitness costs [2] $\square$. The incidence of editing can be regulated in a tissue- or stage-specific manner. For example, A-to-I 
editing in D. melanogaster is enriched in brains and adult tissues $[13,14] \square$, and it exhibits neuron

76 type-specific profiles [15] $\square$. Editing is also responsive to environmental cues, e.g. the response to temperature acclimation in D. melanogaster $[16,17] \square$.

78 If editing is linked to adaptive evolution, it should leave genetic footprints in the genome that can be detected by comparative analyses. Indeed, non-synonymous A-to-I sites in brain transcriptomes are

80 frequently conserved and under positive selection across the Drosophila genus [18,19*,20*] $\square$. Interestingly, phylogenetic comparisons of editing in individual insect genes show that, as

82 hypothesised [2] $\square$, it expands phenotypic space by introducing sequence variation into highly conserved or invariant loci [21] $\square$, or — more subtly — in variable regions within highly conserved

84 genes, e.g. potassium voltage-gated channels [22] $\square$. These diversifying effects can be especially significant in neuronal genes that tend to evolve under strong functional constraints $\left[20^{*}\right] \square$, such as

86 insect nicotinic acetylcholine receptors in which RNA editing provides substantial diversity [23] $\square$.

Adaptive editing can also be studied from a population genetic perspective. For example,

88 evolutionarily recent A-to-I sites in rhesus macaques are more common than expected in loci with recent G-to-A mutations (relative to humans) for both fixed and currently polymorphic loci, and

90 these novel A-to-I sites are under positive selection across macaque populations [24] $\square$. These results suggest that A-to-I compensates the costs of recent G-to-A mutations, a view also supported

92 by detailed analyses of editing conservation in insect nicotinic acetylcholine receptors [23] $\square$. In contrast, Popitsch et al. [25*] $\square$ reported that the adaptiveness of A-to-I in human and $D$.

94 melanogaster populations was due to higher relative fitness of $\mathrm{G}$ alleles in these sites, which A-to-I effectively mimics. These conflicting hypotheses, which can be tested with transcriptomic and

96 population genetic methods, imply that different natural selection mechanisms could be acting on editing sites $\left[25^{*}\right] \square$.

\section{RNA editing is a source of environmental adaptation in insects}

A recent study by Yablonovitch et al. [26**] $\square$ provides strong support for the relationship between

100 editing, adaptation, and fine-scale population genetic diversity. Several editing events were associated with aridity tolerance in D. melanogaster from opposite slopes of the 'Evolution

102 Canyon', near Israel's Mount Carmel, which show dramatic microclimatic differences. The study combined whole-genome sequencing, RNA-seq, and microfluidics-based multiplex PCR (a high-

104 throughput assay to measure allele-specific transcript frequencies [27*] $\square$ ) (Box 1) to investigate the role of DNA mutations in regulating gene expression and the frequency of A-to-I editing in flies 
originating from opposite slopes of the canyon.

Fine-scale population structure in the 'Evolution Canyon' flies correlated with transcriptomic 108 regulation both at the editing and expression levels. Furthermore, differentially edited A-to-I sites were frequently associated with highly-differentiated DNA polymorphisms in their editing

110 complementary sequences (the region in dsRNA molecules that is required for ADAR-mediated Ato-I deamination); and the genomic regions surrounding differential editing sites had stronger interpopulation differentiation than those of constitutive editing sites (Figure 2). A CRISPR-Cas9 mutagenic assay was used to demonstrate the effect of DNA variation in editing rates for the

114 prominin gene, in which an intronic polymorphism exclusive to the north-facing population hindered dsRNA stability and resulted in lower editing rates.

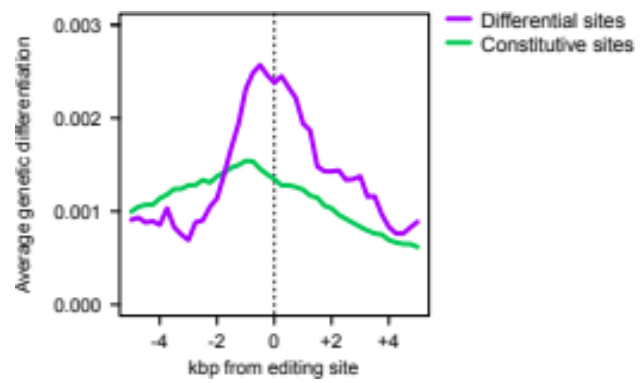

Figure 2. Genetic differentiation around A-to-I editing sites between $D$. melanogaster populations collected from south-facing (arid) and northfacing (humid) slopes in the 'Evolution Canyon'. Differentiation is higher in A-to-I sites that are differentially edited between the two populations (purple) than in constitutive sites (green), reflecting slope-specific regulatory polymorphisms. Figure adapted from Yablonovitch et al. 2017 [26**], with permission from the authors, and reproduced under a Creative Commons Attribution 4.0 International License (creativecommons.org/licenses/by/4.0).

The link between genetic and editing variation is based on the assumption that ADAR activity is

118 regulated by genetically-encoded signals $\left[20^{*}\right] \square$. Current evidence suggests that multiple cisregulatory factors influence editing, such as sequence motifs in A-to-I sites (depletion/enrichment of guanosines upstream/downstream of adenosine sites $[16,28] \square$ ) and their complementary sequences (e.g. cytosines opposite to the editing site increase dsRNA stability and facilitate ADAR activity

$122[21,29] \square)$. A study of D. melanogaster polymorphisms with quantitative effects on A-to-I frequency showed that regulatory loci are located close to (but not overlapping) editing sites, and influence

124 editing frequency by altering dsRNA stability [30] $\square$. Interspecific variation in editing frequency is also influenced by sequence conservation in the D. melanogaster/D. sechellia species pair [31] $\square$;

126 and functionally relevant, conserved editing sites in Drosophila are often under positive selection $[18,19] \square$. 
128 Yet, editing can also be influenced by environmental factors such as temperature. In $D$. melanogaster, A-to-I editing is more common at lower temperatures because ADAR enzymes are

130 more active $[14,17] \square$, recognise dsRNA motifs with higher specificity [16] $\square$, and dsRNAs are more stable $[16,17] \square$. The relative importance of cis-regulatory and environmental factors was

132 investigated by Yablonovitch et al. [26**] $\square$, who found that genetic effects were site-specific and stronger than environmental factors; whereas temperature increases had broad, unspecific effects by

134 virtue of globally reduced editing rates.

\section{RNA editing regulates the activity of insecticide target site proteins}

136 Whilst genome- and transcriptome-wide analyses of RNA editing remain restricted to few taxa, there have been several studies focusing on individual genes and species, with a particular focus on

138 neural ion channels whose kinetics can be fine-tuned by editing-mediated substitutions [32] $\square$. Crucially, many ion channels where functional editing has been described are also target sites of

140 insecticides [33,34] $\square$ - for example, $\gamma$-aminobutyric acid receptors (GABA) [15,35] $\square$, subunits of the nicotinic acetylcholine receptors (nAChR) $[15,36,37] \square$, or voltage-gated sodium channels

142 (VGSC) $[15,38] \square$. Given that mutations in target site genes are a major cause of rising insecticide insensitivity, editing is well-suited to have similar adaptive effects $[33,34] \square$.

$\gamma$-aminobutyric acid receptors (GABA receptors)

GABA receptors are targeted by the insecticides dieldrin, fipronil, and ivermectin [39-41] $\square$, an

146 anti-parasitic and insecticidal drug that shows considerable promise for vector control [41] $\square$. EsSalah et al. [42] $\square$ characterised an editing event near the GABA binding site in Drosophila

$148(R 122 G)$ that decreased its sensitivity to the GABA neurotransmitter and fipronil. Rather than creating a resistant phenotype, this modification enhanced survival in flies carrying resistance

150 alleles (A301S/A301G and/or T350M, suggesting compensation of fitness costs [39,43] $\square$ ).

A recent study in the mosquito vectors Anopheles gambiae, Culex pipiens and Aedes aegypti

$152\left[44^{* *}\right] \square$ identified new editing sites with effects on insecticide resistance. Specifically, the combination of six non-synonymous editing sites in the A. gambiae receptor $(R 119 G, I 162 \mathrm{~V}, I 176 \mathrm{~V}$,

$154 N 183 G, I 278 \mathrm{~V}, \mathrm{~N} 289 \mathrm{D}$ ) altered the activating and inhibiting potencies of the receptor in presence of GABA and ivermectin. Interestingly, functional editing sites in mosquito vectors were located near,

156 but not overlapping, described $D$. melanogaster sites $\left[44^{* *}\right] \square$. This suggests that, unlike the conserved effects of known resistance mutations (codon 301 or 296 mutations in D. melanogaster

158 or A. gambiae, respectively [43] $\square$ ), the location of editing sites in GABA receptors could more 
species-specific.

160

162

164

166

168

170

\section{Voltage-gated sodium channels (VGSC)}

172 VGSCs are the target site of pyrethroids and DDT [50] $\square$. Many base substitutions that reduce the channel sensitivity (knock-down resistance mutations, $k d r$ ) have been identified in insects,

174 including disease vectors [50] $\square$. Initial reports of links between editing-mediated $k d r$ substitutions and pyrethroid resistance in the mosquitoes Culex quinquefasciatus, Aedes albopictus, the house fly Musca domestica, and the cockroach Blatella germanica [51-53] $\square$ were later attributed to methodological errors [54] $\square$, which we speculate may have discouraged further investigations into

178 RNA editing in vectors. Nevertheless, there is independent evidence of non-synonymous editing effecting changes in voltage dependence of activation/inactivation in B. germanica (A-to-I: K184R and I1663M; C-to-U: L1285P and V1685A) [55] $\square$ and D. melanogaster (A-to-I: I260V) [38] $\square$.

\section{Conclusions}

182 Genome-wide investigations of RNA editing in insect vectors have been, to date, noticeably lacking, preventing informed assessment of heir aggregate importance in generating phenotypic

184 diversity. However, evidence from D. melanogaster suggests that this is a fertile line of inquiry for at least two medically-relevant phenotypes: environmental adaptations, and insecticide resistance.

186 There are multiple paths leading from RNA editing to adaptive evolution, each of them with distinct phylogenetic $\left[19^{*}, 20^{*}\right] \square$ and population genetic footprints $\left[25^{*}\right] \square$ that can be detected in cis-

188 regulatory motifs governing editing rates $\left[20^{*}, 26^{* *}, 30,31\right] \square$. Yablonovitch et al. [26**] $\square$ provide a 
blueprint for joint surveys of fine-scale genomic and transcriptomic variation in insects, a path to

190 validate causal links between both, and valuable evidence of overlooked adaptive cis-regulatory changes.

192 Future investigations in vectors should go beyond single-gene approaches $\left[35,44^{* *}\right] \square$ and leverage existing population and comparative genomic resources $[56,57] \square$ to elucidate the dominant

194 mechanisms of evolution of RNA editing in a wider selection of species, and identify regulatory polymorphisms involved in adaptive evolution in natural vector populations. Transcriptome-wide

196 analyses can also expand the range of editing candidate genes to include, for example, enzymes involved in metabolic insecticide resistance [58] $\square$, which have not been usually covered by target-

198 gene approaches. Furthermore, it has recently become possible to investigate the cell type specificity of RNA editing using full-transcript single cell transcriptomic approaches $[59,60] \square$,

200 which can provide fine-grained insights on its functional effects - including resistance adaptations - and possibly inform the development of novel insecticides. Insect disease vectors

202 have remarkable capacity to rapidly evolve and evade control, and going beyond focus on DNA substitutions to understand the range of contributory mechanisms is a key step for the vector 204 genomics community. 


\section{Box 1 - Methods for genome-wide identification of RNA editing sites}

Genome-wide scans of RNA editing sites can be performed using high-throughput sequencing approaches, often based on the fact that inosine bases are incorporated as guanosines by the reverse transcriptases used in RNA-sequencing protocols $[1,3] \square$.

210 RNA editing detection methods based on RNA-seq (see [61] $\square$ for a detailed review) require two steps: (i) RNA-to-genome mapping to identify transcript variants, and (ii) a series of filters aimed at

212 discriminating between editing sites and other sources of polymorphism, such as genomicallyencoded variants (SNPs) and sequencing errors [62,63] (Figure 3). A common solution to filter

214 out genomic variants is the use of paired WGS and RNA-seq experiments from the same sample, under the assumption that variants present in RNA but not in the DNA reads will result from editing

216 (suitable tools include JACUSA [62] $\square$, RES-Scanner [64] $\square$, or reditools [65] $\square$ ).

Less costly procedures based on RNA-seq alone can discriminate editing sites from SNPs by

218 filtering out genomic variants from pre-compiled databases, complete [66] $\square$ or partial (GIREMI $[67] \square)$. Other tools discriminate between editing sites and SNPs by taking advantage of the

220 tendency of editing to occur in hyper-editing clusters ([68] $\square$, SPRINT [69] $\square)$.

Any analysis of RNA editing might also benefit from a comparative perspective - i.e., differential

222 editing between insect populations, tissues, or biological conditions, etc. In that respect, all the above-mentioned methods provide per-site editing frequencies that can be compared ad-hoc, and

224 some are able to perform explicit differential analyses (JACUSA [62] $\square$ ).

Finally, the microfluidics-based multiplex PCR (mmPCR-seq) is a general approach to measure

226 transcript allelic ratios, including editing events $\left[27^{*}\right] \square$. This high-throughput method requires prior knowledge of the sites, but it enables the estimation of editing rates at higher accuracy than

228 RNA-seq. It has been used to investigate population- $\left[26^{* *}\right] \square$ and tissue-specific $[15,20] \square$ editing profiles in D. melanogaster. 


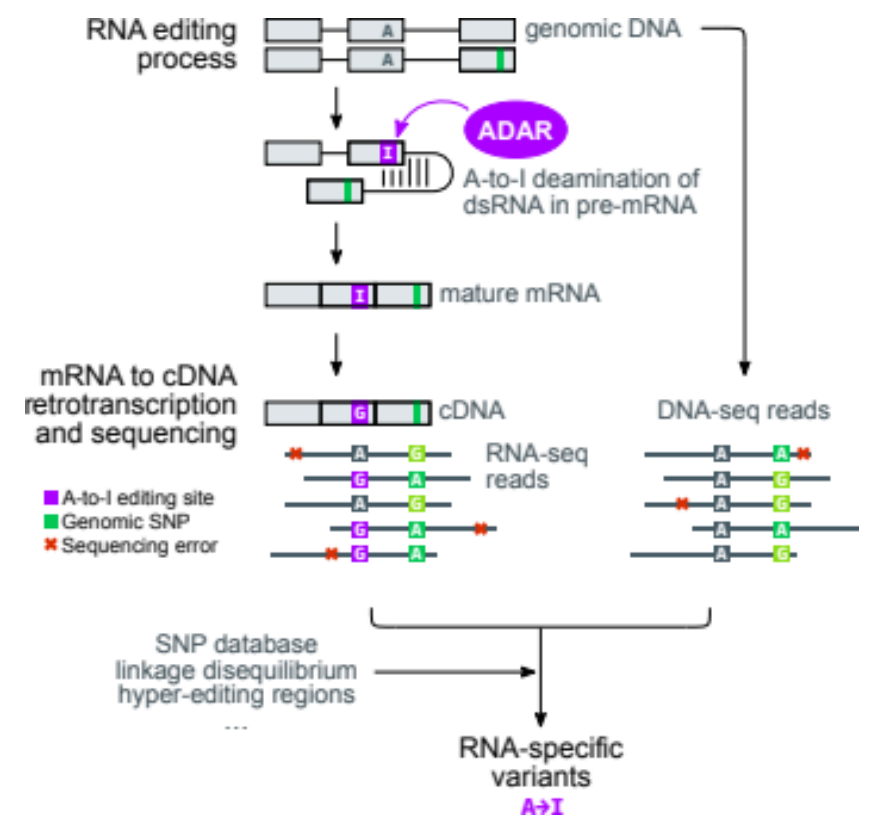

Figure 3. Summary of a high-throughput approach to detect RNA editing events, based on paired RNA-seq and DNA-seq experiments. A-to-I editing is used as an example. 


\section{Key references}

Duan et al. 2017 [19*] $\square$. Investigation of A-to-I editing in neural tissues in closely-related

234 Drosophila species. The authors demonstrate that editing is enriched in neural tissues and affects functionally constrained genes, and highlight the adaptive value of conserved editing sites in

236 insects.

Zhang et al. 2017 [20*] $\square$. Using comparative transcriptomic and genomic analyses of multiple

238 Drosophila species, the authors demonstrate the importance of the cis-regulatory landscape in regulating editing variation. The authors also trace gains and losses of editing sites across species,

240 and show that widely-conserved sites are enriched in slow-evolving neural genes.

Popitsch et al. 2017 [25*] $\square$. Investigation of the population-genetic footprints underpinning the

242 evolution of adaptive editing. The authors provide a comprehensive list of hypotheses with testable predictions. They find support for an adaptive role of A-to-I editing as a transcriptomic 'mimicry' of

244 adaptive A-to-G mutations in both D. melanogaster and humans.

Yablonovitch et al. 2017 [26**] $\square$. The authors use a combination of WGS, RNA-seq and targeted

246 assays to unravel the role of A-to-I editing in two closely related populations of D. melanogaster with divergent climatic adaptations. They are able to link population genetic divergence to

248 regulatory variation in editing, and they identify candidate genes for validation.

Zhang et al. $2014\left[27^{*}\right] \square$. The authors propose a new high-throughput assay to measure allelic

250 ratios in transcripts at high precision, which can be coupled with genomic and transcriptomic analyses to RNA editing variants.

252 Taylor-Wells et al. 2018 [44**] $\square$. This ground-breaking study demonstrates that multiple editing events in the GABA receptor of A. gambiae can change the electrophysical properties of the

254 channel, and result in reistane to ivermectin. The authors also study the evolutionary conservation of the mutations in other vectors and $D$. melanogaster.

256 Rinkevich et al. 2012 [49*] $\square$. The authors demonstrate that ADAR-defective D. melanogaster are more susceptible to insecticides that target the heavily edited nicotinic acetylcholine receptors. 
1. Nishikura K: A-to-I editing of coding and non-coding RNAs by ADARs. Nat Rev Mol Cell Biol 2016, 17:83-96.

2. Gommans WM, Mullen SP, Maas S: RNA editing: a driving force for adaptive evolution? Bioessays 2009, 31:1137-1145.

3. Eisenberg E, Levanon EY: A-to-I RNA editing - Immune protector and transcriptome diversifier. Nat Rev Genet 2018, 19:473-490.

4. Savva YA, Rieder LE, Reenan RA: The ADAR protein family. Genome Biol 2012, 13:252.

5. Li Q, Zhang P, Li J, Yu H, Zhan X, Zhu Y, Guo Q, Tan H, Lundholm N, Garcia L, et al.: On the origin and evolution of RNA editing in metazoans. bioRxiv 2020, doi:10.1101/2020.01.19.911685.

6. Blanc V, Davidson NO: APOBEC-1-mediated RNA editing. Wiley Interdiscip Rev Syst Biol Med 2010, 2:594-602.

7. Gerber AP, Keller W: RNA editing by base deamination: more enzymes, more targets, new mysteries. Trends Biochem Sci 2001, 26:376-384.

8. Yablonovitch AL, Deng P, Jacobson D, Li JB: The evolution and adaptation of A-to-I RNA editing. PLOS Genet 2017, 13:e1007064.

9. Solomon O, Oren S, Safran M, Deshet-Unger N, Akiva P, Jacob-Hirsch J, Cesarkas K,

10. Hsiao Y-HE, Bahn JH, Yang Y, Lin X, Tran S, Yang E-W, Quinones-Valdez G, Xiao X: RNA editing in nascent RNA affects pre-mRNA splicing. Genome Res 2018, 28:812-823.

280 11. Mazloomian A, Meyer IM: Genome-wide identification and characterization of tissuespecific RNA editing events in D. melanogaster and their potential role in regulating alternative splicing. RNA Biol 2015, 12:1391-1401.

12. Ekdahl Y, Farahani HS, Behm M, Lagergren J, Öhman M: A-to-I editing of microRNAs in the mammalian brain increases during development. Genome Res 2012, 22:1477-1487.

13. Palladino MJ, Keegan LP, O'Connell MA, Reenan RA: A-to-I Pre-mRNA Editing in 2000, 102:437-449.

288 14. Savva YA, Jepson JEC, Sahin A, Sugden AU, Dorsky JS, Alpert L, Lawrence C, Reenan RA: Auto-regulatory RNA editing fine-tunes mRNA re-coding and complex behaviour in Drosophila. Nat Commun 2012, 3.

15. Sapiro AL, Shmueli A, Henry GL, Li Q, Shalit T, Yaron O, Paas Y, Li JB, Shohat-Ophir G: 
Natl Acad Sci 2019, doi:10.1073/PNAS.1811768116.

294 16. Buchumenski I, Bartok O, Ashwal-Fluss R, Pandey V, Porath HT, Levanon EY, Kadener S: Dynamic hyper-editing underlies temperature adaptation in Drosophila. PLOS Genet 2017, 13:e1006931.

17. Rieder LE, Savva YA, Reyna MA, Chang Y-J, Dorsky JS, Rezaei A, Reenan RA: Dynamic 298 response of RNA editing to temperature in Drosophila. BMC Biol 2015, 13:1.

18. Yu Y, Zhou H, Kong Y, Pan B, Chen L, Wang H, Hao P, Li X: The Landscape of A-to-I 300 RNA Editome Is Shaped by Both Positive and Purifying Selection. PLOS Genet 2016, 12:e1006191.

19. Duan Y, Dou S, Luo S, Zhang H, Lu J: Adaptation of A-to-I RNA editing in Drosophila. PLOS Genet 2017, 13:e1006648.

20. Zhang R, Deng $\mathrm{P}$, Jacobson $\mathrm{D}$, Li JB: Evolutionary analysis reveals regulatory and functional landscape of coding and non-coding RNA editing. PLOS Genet 2017, 13:e1006563.

21. Reenan RA: Molecular determinants and guided evolution of species-specific RNA editing. Nature 2005, 434:409-413.

22. Yang Y, Lv J, Gui B, Yin H, Wu X, Zhang Y, Jin Y: A-to-I RNA editing alters lessconserved residues of highly conserved coding regions: implications for dual functions in evolution. $R N A$ 2008, 14:1516-1525.

23. Tian $\mathrm{N}, \mathrm{Wu} \mathrm{X}$, Zhang $\mathrm{Y}$, Jin $\mathrm{Y}$ : A-to-I editing sites are a genomically encoded G: implications for the evolutionary significance and identification of novel editing sites. RNA 2008, 14:211-216.

24. An NA, Ding W, Yang X-Z, Peng J, He BZ, Shen QS, Lu F, He A, Zhang YE, Tan BC-M, et

25. Popitsch N, Huber CD, Buchumenski I, Eisenberg E, Jantsch M, Haeseler A von, Gallach M: A-to-I RNA editing uncovers hidden signals of adaptive genome evolution in animals. bioRxiv 2017, doi:10.1101/228734.

26. Yablonovitch AL, Fu J, Li K, Mahato S, Kang L, Rashkovetsky E, Korol AB, Tang H, Michalak P, Zelhof $\mathrm{AC}$, et al.: Regulation of gene expression and RNA editing in Drosophila adapting to divergent microclimates. Nat Commun 2017, 8:1570.

27. Zhang R, Li X, Ramaswami G, Smith KS, Turecki G, Montgomery SB, Li JB: Quantifying RNA allelic ratios by microfluidic multiplex PCR and sequencing. Nat Methods 2014, 11:51-54.

28. Eggington JM, Greene T, Bass BL: Predicting sites of ADAR editing in double-stranded RNA. Nat Commun 2011, 2. 
29. Matthews MM, Thomas JM, Zheng Y, Tran K, Phelps KJ, Scott AI, Havel J, Fisher AJ, Beal PA: Structures of human ADAR2 bound to dsRNA reveal base-flipping mechanism and basis for site selectivity. Nat Struct Mol Biol 2016, 23:426-433.

332 30. Kurmangaliyev YZ, Ali S, Nuzhdin S V.: Genetic Determinants of RNA Editing Levels of ADAR Targets in Drosophila melanogaster. G3 Genes, Genomes, Genet 2016, 6:391-396.

334 31. Sapiro AL, Deng P, Zhang R, Li JB: Cis Regulatory Effects on A-to-I RNA Editing in Related Drosophila Species. Cell Rep 2015, 11:697-703.

336 32. Rosenthal JJC, Seeburg PH: A-to-I RNA editing: effects on proteins key to neural excitability. Neuron 2012, 74:432-9.

338 33. ffrench-Constant RH, Williamson MS, Davies TGE, Bass C: Ion channels as insecticide targets. J Neurogenet 2016, 30:163-177.

340 34. Jones AK: Genomics, cys-loop ligand-gated ion channels and new targets for the control of insect pests and vectors. Curr Opin Insect Sci 2018, 30:1-7.

342 35. Jones AK, Buckingham SD, Papadaki M, Yokota M, Sattelle BM, Matsuda K, Sattelle DB: Splice-Variant- and Stage-Specific RNA Editing of the Drosophila GABA Receptor Modulates Agonist Potency. J Neurosci 2009, 29:4287-4292.

36. Grauso M, Reenan RA, Culetto E, Sattelle DB: Novel putative nicotinic acetylcholine receptor subunit genes, Dalpha5, Dalpha6 and Dalpha7, in Drosophila melanogaster identify a new and highly conserved target of adenosine deaminase acting on RNAmediated A-to-I pre-mRNA editing. Genetics 2002, 160:1519-33.

37. Hoopengardner B, Bhalla T, Staber C, Reenan R: Nervous System Targets of RNA Editing Identified by Comparative Genomics. Science (80- ) 2003, 301:832-836.

38. Olson ROD, Liu Z, Nomura Y, Song W, Dong K: Molecular and functional characterization of voltage-gated sodium channel variants from Drosophila melanogaster. Insect Biochem Mol Biol 2008, 38:604-610.

354 39. ffrench-Constant RH, Rocheleau TA, Steichen JC, Chalmers AE: A point mutation in a Drosophila GABA receptor confers insecticide resistance. Nature 1993, 363:449-451.

40. Gant DB, Chalmers AE, Wolff MA, Hoffman HB, Bushey D: Fipronil: action at the GABA receptor. In Pesticides and the Future. Edited by Kuhr RJ, Motoyama N. IOS Press; 1998:147-156.

41. Chaccour C, Hammann F, Rabinovich NR: Ivermectin to reduce malaria transmission I. Pharmacokinetic and pharmacodynamic considerations regarding efficacy and safety. Malar J 2017, 16.

362 42. Es-Salah Z, Lapied B, Le Goff G, Hamon A: RNA editing regulates insect gammaaminobutyric acid receptor function and insecticide sensitivity. Neuroreport 2008, 19:939-943. 
43. Thompson M, Steichen JC, ffrench-Constant RH: Conservation of cyclodiene insecticide 366 resistance-associated mutations in insects. Insect Mol Biol 1993, 2:149-154.

44. Taylor-Wells J, Senan A, Bermudez I, Jones AK: Species specific RNA A-to-I editing of mosquito RDL modulates GABA potency and influences agonistic, potentiating and antagonistic actions of ivermectin. Insect Biochem Mol Biol 2018, 93:1-11.

45. Dripps JE, Boucher RE, Chloridis A, Cleveland CB, DeAmicis C V., Gomez LE, Paroonagian DL, Pavan LA, Sparks TC, Watson GB: CHAPTER 5. The Spinosyn Insecticides. 2011:163-212.

46. Matsuda K, Buckingham SD, Kleier D, Rauh JJ, Grauso M, Sattelle DB: Neonicotinoids: Insecticides acting on insect nicotinic acetylcholine receptors. Trends Pharmacol Sci 2001, 22:573-580.

47. Sattelle DB, Jones AK, Sattelle BM, Matsuda K, Reenan R, Biggin PC: Edit, cut and paste in the nicotinic acetylcholine receptor gene family of Drosophila melanogaster. Bioessays 2005, 27:366-376.

48. Yao X, Song F, Zhang Y, Shao Y, Li J, Liu Z: Nicotinic acetylcholine receptor $\beta 1$ subunit

49. Rinkevich FD, Scott JG: Reduction of dADAR activity affects the sensitivity of Drosophila melanogaster to spinosad and imidacloprid. Pestic Biochem Physiol 2012, 104:163-169.

50. Dong K, Du Y, Rinkevich F, Nomura Y, Xu P, Wang L, Silver K, Zhorov BS: Molecular biology of insect sodium channels and pyrethroid resistance. Insect Biochem Mol Biol 2014, 50:1-17.

51. $\mathrm{Xu} \mathrm{Q}$, Wang $\mathrm{H}$, Zhang $\mathrm{L}$, Liu N: Sodium channel gene expression associated with pyrethroid resistant house flies and German cockroaches. Gene 2006, 379:62-67.

52. $\mathrm{Xu} \mathrm{Q}$, Wang $\mathrm{H}$, Zhang $\mathrm{L}$, Liu $\mathrm{N}$ : Kdr allelic variation in pyrethroid resistant mosquitoes, Culex quinquefasciatus (S.). Biochem Biophys Res Commun 2006, 345:774-780.

53. Liu N, Xu Q, Zhang L: Sodium channel gene expression in mosquitoes, Aedes albopictus (S.). Insect Sci 2006, 13:431-436.

54. Donnelly MJ, Corbel V, Weetman D, Wilding CS, Williamson MS, Black IV WC: Does kdr genotype predict insecticide-resistance phenotype in mosquitoes? Trends Parasitol 2009, 25:213-219.

55. Song W, Liu Z, Tan J, Nomura Y, Dong K: RNA Editing Generates Tissue-specific Sodium Channels with Distinct Gating Properties. J Biol Chem 2004, 279:32554-32561.

56. Miles A, Harding NJ, Bottà G, Clarkson CS, Antão T, Kozak K, Schrider DR, Kern AD, 
Anopheles gambiae. Nature 2017, 552:96-100.

402 57. Neafsey DE, Waterhouse RM, Abai MR, Aganezov SS, Alekseyev MA, Allen JE, Amon J, Arcà $B$, Arensburger $P$, Artemov $G$, et al.: Highly evolvable malaria vectors: The genomes 404 of 16 Anopheles mosquitoes. Science (80- ) 2015, 347:1258522.

58. Li X, Schuler MA, Berenbaum MR: Molecular mechanisms of metabolic resistance to 406 synthetic and natural xenobiotics. Annu Rev Entomol 2007, 52:231-253.

59. Picardi E, Horner DS, Pesole G: Single-cell transcriptomics reveals specific RNA editing 408 signatures in the human brain. RNA 2017, 23:860-865.

60. Qiu S, Li W, Xiong H, Liu D, Bai Y, Wu K, Zhang X, Yang H, Ma K, Hou Y, et al.: Singlecell RNA sequencing reveals dynamic changes in A-to-I RNA editome during early human embryogenesis. BMC Genomics 2016, 17:766.

61. Diroma MA, Ciaccia L, Pesole G, Picardi E: Elucidating the editome: bioinformatics approaches for RNA editing detection. Brief Bioinform 2017, doi:10.1093/bib/bbx129.

62. Piechotta M, Wyler E, Ohler U, Landthaler M, Dieterich C: JACUSA: site-specific identification of RNA editing events from replicate sequencing data. BMC Bioinformatics 2017, 18:7.

63. John D, Weirick T, Dimmeler S, Uchida S: RNAEditor: easy detection of RNA editing events and the introduction of editing islands. Brief Bioinform 2017, 18:993-1001.

64. Wang Z, Lian J, Li Q, Zhang P, Zhou Y, Zhan X, Zhang G: RES-Scanner: a software 420 package for genome-wide identification of RNA-editing sites. Gigascience 2016, 5:37.

65. Picardi E, D’Erchia AM, Montalvo A, Pesole G: Using REDItools to Detect RNA Editing Events in NGS Datasets. In Current Protocols in Bioinformatics. . John Wiley \& Sons, Inc.; 2015:12.12.1-12.12.15.

424 66. Ramaswami G, Zhang R, Piskol R, Keegan LP, Deng P, O’Connell MA, Li JB: Identifying RNA editing sites using RNA sequencing data alone. Nat Methods 2013, 10:128-132.

426 67. Zhang Q, Xiao X: Genome sequence-independent identification of RNA editing sites. Nat Methods 2015, 12:347-350.

428 68. Porath HT, Carmi S, Levanon EY: A genome-wide map of hyper-edited RNA reveals numerous new sites. Nat Commun 2014, 5:4726.

430 69. Zhang F, Lu Y, Yan S, Xing Q, Tian W: SPRINT: An SNP-free toolkit for identifying RNA editing sites. Bioinformatics 2017, 33:3538-3548. 